\title{
COVID-19-Versorgung - Strategien der Taskforce Coronavirus und Erfahrungen von den ersten 115 Fällen am Universitätsklinikum Freiburg
}

\section{COVID-19-Response - Strategies of the Task-Force Coronavirus and experiences upon implementation in the management of 115 cases at the University Medical Center Freiburg}

Autoren

Siegbert Rieg1', Hans-Jörg Busch², Felix Hans², Hajo Grundmann³, Paul Biever ${ }^{4}$, Hartmut Bürkle ${ }^{5}$, Thorsten Hammer², Robert Thimme', Winfried Kern ${ }^{1}$

Institute

1 Klinik für Innere Medizin II - Gastroenterologie, Hepatologie, Endokrinologie, Infektiologie, Universitätsklinikum Freiburg, Medizinische Fakultät, Albert-Ludwigs-Universität Freiburg

2 Universitäts-Notfallzentrum (UNZ), Universitätsklinikum Freiburg, Medizinische Fakultät, Albert-LudwigsUniversität Freiburg

3 Institut für Infektionsprävention und Krankenhaushygiene, Universitätsklinikum Freiburg, Medizinische Fakultät, Albert-Ludwigs-Universität Freiburg

4 Universitäts-Herzzentrum Freiburg-Bad Krozingen, Universitätsklinikum Freiburg, Medizinische Fakultät, Albert-Ludwigs-Universität Freiburg

5 Klinik für Anästhesiologie und Intensivmedizin, Universitätsklinikum Freiburg, Medizinische Fakultät, Albert-Ludwigs-Universität Freiburg

Schlüsselwörter

Coronavirus, COVID-19, Komorbidität, Versorgungsstrategien, Behandlung, Überleben

Key words

Coronavirus, COVID-19, comorbidities, models of care, management, survival

Bibliografie

DOI https://doi.org/10.1055/a-1147-6244

Online-Publikation: 28.4.2020

Dtsch Med Wochenschr 2020; 145: 657-664

(c) Georg Thieme Verlag KG, Stuttgart · New York ISSN 0012-0472

Korrespondenzadresse

Prof. Dr. med. Siegbert Rieg

Abteilung Infektiologie, Klinik für Innere Medizin II Universitätsklinikum Freiburg, Medizinische Fakultät Albert-Ludwigs-Universität Freiburg, Hugstetter Straße 55, 79106 Freiburg

Tel.: ++ 49/7 61/27018190

Fax: $++49 / 761 / 27018200$

siegbert.rieg@uniklinik-freiburg.de

\section{ZUSAMMENFASSUNG}

Hintergrund Das neue, pandemische Coronavirus SARS-CoV-2 und die damit einhergehende Erkrankung COVID-19 (Coronavirus Disease 2019) stellt die medizinischen Versorgungssysteme vor große Herausforderungen. Die vorliegende Arbeit fasst die Strategie, Organisation und Maßnahmen der COVIDTaskforce des Universitätsklinikums Freiburg zusammen und berichtet über Erfahrungen und Behandlungsergebnisse von der Versorgung der ersten 115 COVID-19-Patienten.

Methoden Retrospektive, narrative Prozessbeschreibung und -analyse des Zeitraums Ende Januar bis Anfang April 2020, durchgeführt unter Mitwirken der beteiligten Departments, Kliniken und Institute des Universitätsklinikums Freiburg. Ergänzend erfolgte eine retrospektive Beobachtungsstudie mit deskriptiver statistischer Auswertung der epidemiologischen und klinischen Daten aller bis 31. März 2020 hospitalisierten COVID-19-Patienten.

Ergebnisse Durch eine interdisziplinär zusammengesetzte Taskforce Coronavirus wurden Maßnahmen in den Bereichen ambulante Testung und Beratung, Separierung der Patientenströme sowie Eskalation der Versorgungskapazitäten auf Normal- und Intensivstationen im Sinne eines dynamischen Stufenmodells angeregt und umgesetzt. Die Versorgung der ersten 115 Patienten gelang somit ohne Entstehung von Engpässen.

Diskussion Durch Zusammenwirken sämtlicher Disziplinen und Abteilungen des Universitätsklinikums Freiburg konnte eine adäquate Versorgung von COVID-19-Patienten sichergestellt und die Handlungsfähigkeit des Klinikums in der aktuellen Ausbruchsituation erhalten werden.

\section{ABSTRACT}

Background The new pandemic coronavirus SARS-CoV-2 causing coronavirus disease-2019 (COVID-19) poses immense challenges to health care systems worldwide. In the current manuscript we summarize the strategies, organisational approaches and actions of the Task-force Coronavirus at the University Medical Center Freiburg. We also report on experiences with implementation of these approaches and treatment outcomes in the first 115 COVID patients. 
Methods Retrospective, narrative process description and analysis of the time period between end of January and beginning of April 2020, performed by representatives of the involved departments and institutes. Additionally a retrospective observational cohort study with descriptive analysis of epidemiological and clinical data of COVID patients admitted until March 31st was performed.

Results A multidisciplinary Task-force Coronavirus initiated measures concerning outpatient testing and counseling, reorganisation and separation of patient flow processes alongside with substantial escalation of inpatient capacities on regular wards and intensive care units. Within the framework of the resulting dynamic care model, 115 patients suffering from COVID could be treated without shortages in staff or bed capacities.

Dicussion In the upcoming pandemic, adequate COVID management and care could be secured by a collaborative approach with inclusion of administrative departments, clinical disciplines and theoretical institutes of the University Medical Center Freiburg.

\section{Einleitung}

Aus dem Ende 2019 regional aufgetretenen Ausbruch schwerer respiratorischer Infektionen durch das neue Coronavirus SARSCoV-2 hat sich rasch eine weltweite Pandemie entwickelt [1]. In Europa waren früh Norditalien, konsekutiv die Schweiz und das östliche Frankreich betroffen. Aufgrund der räumlichen Nähe und des regen Grenzverkehrs kam es in Südbaden rasch zu hohen COVID-19 (Coronavirus Disease 2019) -Erkrankungszahlen [2]. In der vorliegenden Arbeit werden die Strategie und die Organisationsstrukturen des Universitätsklinikums Freiburg als Antwort auf die SARS-CoV-2-Pandemie vorgestellt. Darüber hinaus wird die Implementierung bzw. praktische Umsetzung anhand Erfahrungen und Behandlungsergebnissen der ersten 115 COVID-19Patienten evaluiert und diskutiert.

\section{Methoden}

Retrospektive, narrative Prozessbeschreibung und -analyse unter Mitwirken von Beteiligten der klinischen Disziplinen/Abteilungen und theoretischen Institute. Zudem erfolgte eine Sichtung der Protokolle der Taskforce Coronavirus sowie ein Abgleich mit den Meldungen der innerbetrieblichen Kommunikationsplattform im Intranet des Universitätsklinikums Freiburg.

Zur Analyse der COVID-19-Patienten wurden epidemiologische und klinische Daten, Befunde der Virologie/Mikrobiologie, Therapie und Behandlungsdaten erhoben. Eingeschlossen wurden hospitalisierte Patienten mit Vorliegen einer SARS-CoV-2-positiven PCR aus respiratorischem Material (Rachenabstrich, Trachealoder Bronchialsekret/-lavage). Ein positives Votum der Ethikkommission des Universitätsklinikums Freiburg zur anonymisierten, retrospektiven Datenerhebung und Auswertung liegt vor.

\section{Ergebnisse}

Am Universitätsklinikum Freiburg formierte sich im Verlauf des Januars eine Arbeitsgruppe Coronavirus, die sich, in Fortsetzung der Arbeitsgruppe Ebolavirus aus dem Jahr 2014, aus Mitgliedern der Abteilung Infektiologie, des Universitäts-Notfallzentrums (UNZ) und des Instituts für Umweltmedizin und Krankenhaushygiene zusammensetzte. Aus dieser Kernarbeitsgruppe entwickelte sich unter Einbeziehung von Vertretern des Instituts für Viro- logie, des Departments Innere Medizin (inkl. Intensivmedizin), der Klinik für Anästhesiologie und Intensivmedizin, der Pflegedienstleitung und Klinikdirektion sowie Vertretern des Gesundheitsamtes Freiburg die Taskforce Coronavirus, die ab Ende Februar mehrmals wöchentlich zu Planungstreffen zusammentraf. Die Taskforce Coronavirus beriet und informierte im weiteren Verlauf die Klinikeinsatzleitung, der nach Feststellung des außergewöhnlichen medizinischen Versorgungsnotstands die Koordination und Leitung der COVID-19-Response zufiel.

Im Folgenden werden wesentliche Strategien der beteiligten Bereiche aufgeführt. Die zeitliche Abfolge der eingesetzten Organisationsstrukturen und erhobenen Maßnahmen in Relation zu den steigenden COVID-19-Fallzahlen werden in $\mathbf{A b b} \mathbf{1}$ dargestellt.

\section{Universitäts-Notfallzentrum/ Klinische Akut- und Notfallmedizin}

Durch die Arbeitsgruppe Coronavirus wurde Ende Januar eine Standard Operating Procedure (SOP) zum Vorgehen bei Verdacht auf SARS-CoV-2-Infektion erstellt. Unmittelbar nach Bekanntwerden der ersten COVID-19-Fälle in Baden-Württemberg kam es Ende Februar zu einem signifikanten Anstieg der ambulanten Vorstellungen zur virologischen Diagnostik. Zudem wuchs der telefonische Beratungsbedarf sowohl vonseiten der Patienten als auch der niedergelassenen Ärzteschaft rasch, woraufhin eine Patienteninformations-Hotline (bis zu 500 Anrufe pro Tag) etabliert wurde. Um eine signifikante Beeinträchtigung der allgemeinen Notfallversorgung zu verhindern, wurde die Einrichtung separierter SARS-CoV-2-Abstrichbereiche erforderlich. Bei hohem Patientenaufkommen wurden die Patientenströme ab Anfang März komplett getrennt: Für Patienten mit Verdacht auf COVID19 oder mit respiratorischer Symptomatik wurde ein separates Eingangsportal sowie nachgelagert eine Holding-Station eingerichtet, in der eine Patientenisolierung und ggf. Kohortierung bis zum Erhalt der PCR-Befunde möglich war ( $\mathbf{A} \mathbf{A b}$ b. 2). In enger Absprache richtete die Kassenärztliche Vereinigung Baden-Württemberg im Verlauf ein ambulantes Abstrich-Zentrum im Stadtgebiet ein; dies erbrachte eine Entlastung im Hinblick auf ambulante Testungen.

Für Mitarbeiter wurde in Kooperation mit der Betriebsmedizin ab der 2. Märzwoche ein ausgelagerter SARS-CoV-2-Abstrichbereich etabliert. Neben der Patienten-Hotline für die Bevölkerung wurde auch eine Hotline für Mitarbeiter eingerichtet, die im wei- 


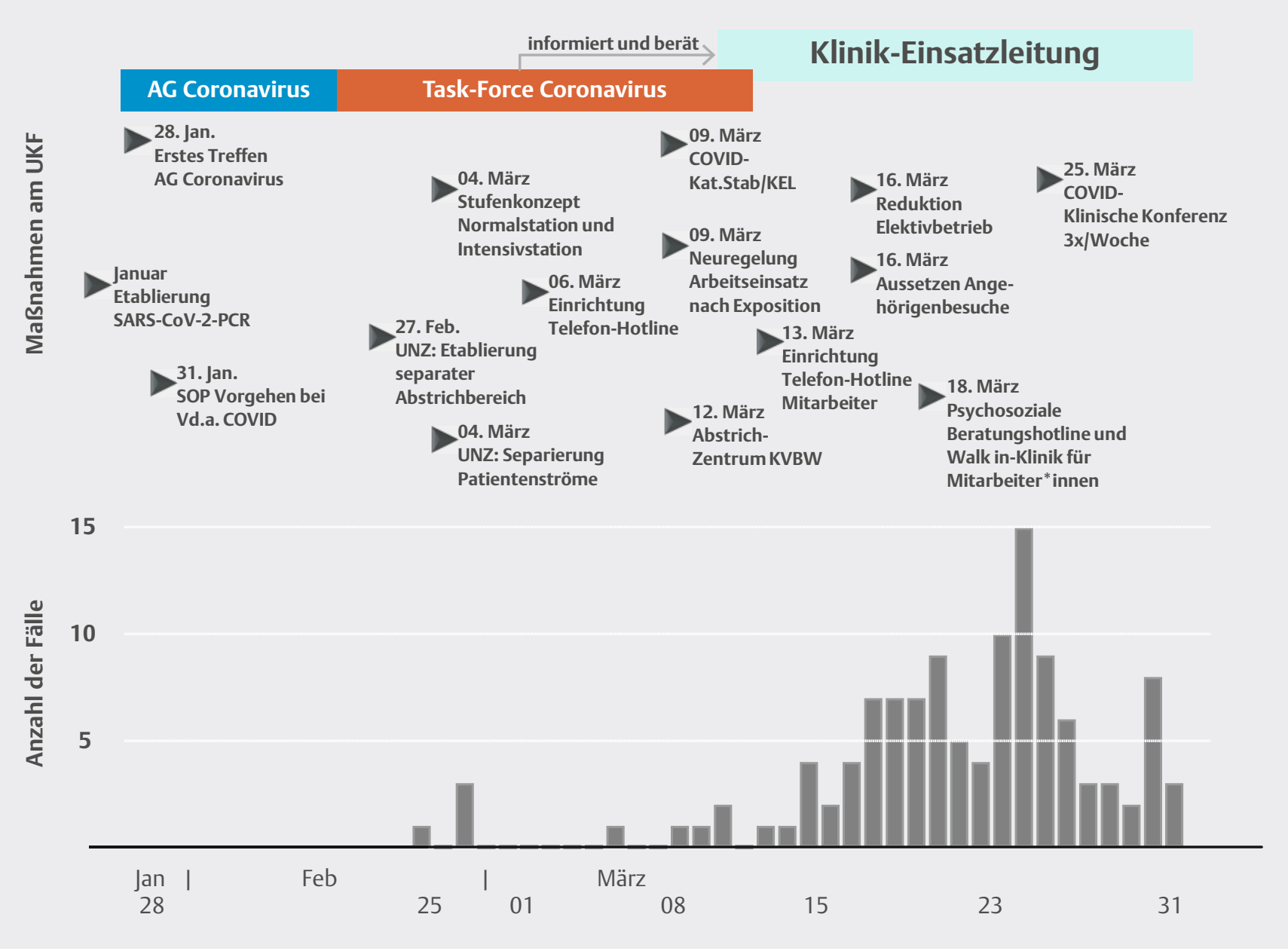

- Abb. 1 COVID-19-Maßnahmen am Universitätsklinikum Freiburg: Organisationsstrukturen und erhobene Maßnahmen in zeitlicher Relation zu COVID-19-Fallzahlen. AG = Arbeitsgruppe; KEL = Klinik-Einsatzleitung; UNZ = Universitäts-Notfallzentrum; KV-BW= Kassenärztliche Vereinigung Baden-Württemberg; SARS-CoV-2 = Severe Acute Respiratory Syndrome-Coronavirus-2.

teren Verlauf um ein psychosoziales Beratungsangebot (Hotline und Walk-in-Klinik) durch die Klinik für Psychosomatische Medizin und Psychotherapie erweitert wurde.

\section{Krankenhaushygiene}

Ausgehend von den zum jeweiligen Zeitpunkt vorliegenden epidemiologischen Daten aus der Lombardei, dem Département Haut-Rhin sowie den Fallzahlen in Stadt und Landkreis wurden Vorhersagemodelle zur erwarteten Erkrankungsinzidenz in der Bevölkerung und der daraus resultierenden Entwicklung des Bettenbedarfs erstellt sowie im Verlauf regelmäßig angepasst. SOPs zu Hygienemaßnahmen wurden in Abhängigkeit von den Modellvorhersagen und auch unter Berücksichtigung der zur Verfügung stehenden Schutzausrüstung (FFP2-Masken und Schutzkittel) fortwährend überprüft und angepasst. Schulungs- und Aufklärungsmaterial wurde mittels Intranets zur Verfügung gestellt. Für die im Verlauf aktivierten COVID-19-Versorgungsbereiche wurden Hygiene-Schulungen vor Ort und regelmäßige Prozessbeobachtungen durchgeführt. Dabei wurden über 1500 Mitarbeiter geschult. Es wurde besonders auf das sachgemäße An- und Ablegen der Schutzbekleidung, auf das Vermeiden von kontamina- tionsträchtigen Handgriffen (Gesicht, Schutzmaske), die korrekte Händehygiene, kollegiales „Nudging“ bei Beobachten von Hygienefehlern und die Bedeutung des prozessbezogenen Anlegens der persönlichen Schutzausrüstung eingegangen.

Strategien der Kontaktpersonen-Nachverfolgung bedurften einer kontinuierlichen Neubewertung. Während in der frühen Phase des Containments (Eindämmung) alle Risikokategorie-I-Kontakte getestet wurden, erfolgte in der späteren Protection (Schutz)Phase eine Testung nur bei symptomatischen Mitarbeitern. Analog wurde die anfängliche Dienstbefreiung von exponiertem Klinikpersonal aufgegeben und im Einvernehmen mit dem Gesundheitsamt Freiburg der Arbeitseinsatz von Exponierten unter Tragen von Mund-Nasen-Schutz erlaubt, solange diese asymptomatisch blieben.

\section{Virologie}

Im Januar wurde die erforderliche Diagnostik mittels SARS-CoV-2PCR etabliert. Bereits Ende Februar war der Ausbau der personellen und technischen Testkapazitäten vordringlich, da mit Anstieg der COVID-19-Fallzahlen die Mitarbeitertestungen nach Exposition deutlich zunahmen. PCR-Testungen wurden um 11 und 


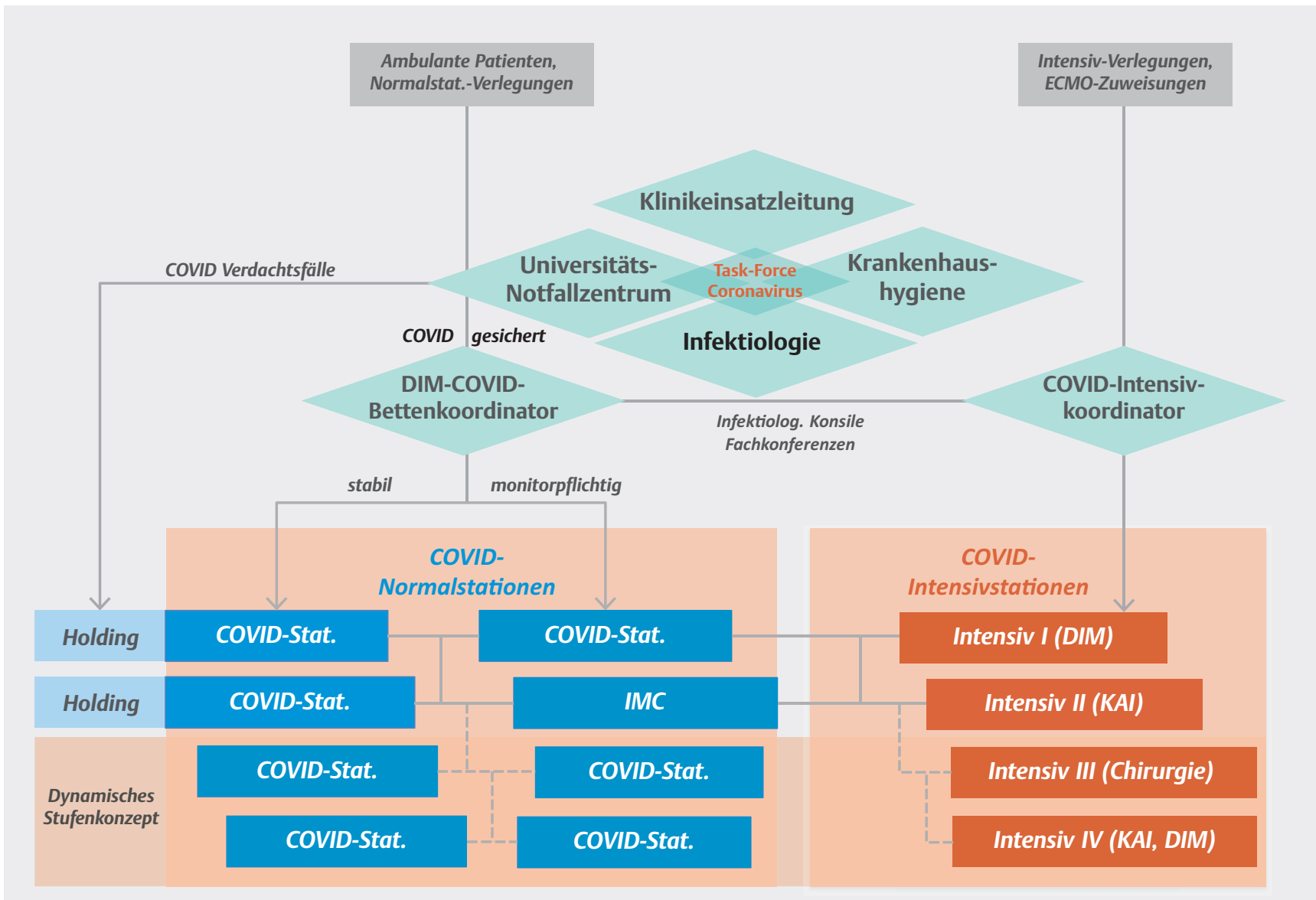

- Abb.2 Patientenflüsse und dynamisches Stufenkonzept zur stationären Versorgung von COVID-19-Patienten. Patientenzuweisungen aus dem ambulanten Bereich wurden im UNZ triagiert und entweder dem Holding-Bereich zugewiesen oder über den Bettenkoordinator des Departments Innere Medizin (DIM) abhängig vom Schweregrad auf eine COVID-19-Station verlegt. Intensivstationsverlegungen oder Zuweisungen zur extrakorporalen Membranoxygenierung (ECMO) wurden über den Intensivkoordinator angenommen und gemäß Verfügbarkeiten den Intensivbereichen zugewiesen. $\mathrm{KAI}=$ Klinik für Anästhesiologie und Intensivmedizin; IMC = Intermediate Care-Station; COVID-19=Coronavirus Disease 2019.

16 Uhr täglich durchgeführt, Testergebnisse lagen jeweils 5 Stunden später vor. Um den Ausfall des Pflege- und Ärztepersonals gering zu halten, mussten zeitweise, basierend auf der Infektionswahrscheinlichkeit, Priorisierungen von stationären Patientenund Mitarbeiter-Abstrichproben vorgenommen werden. Nach entsprechender Absprache wurden die Proben der ambulanten Abstrich-Zentren primär durch niedergelassene Labore untersucht.

\section{COVID-19-Normalstationen}

Nach Aufnahme der ersten COVID-19-Patienten in einen separierten Bereich der Infektionsstation wurde diese ab Anfang März die erste COVID-19-Station des Departments Innere Medizin mit Versorgung von ausschließlich SARS-CoV-2-Infizierten. Gemäß der Fallzahlprognosen wurden konsekutiv in den Departments für Innere Medizin und Chirurgie weitere Stationen vom Normalbetrieb getrennt und als COVID-19-Stationen in Betrieb genommen, gemäß eines dynamischen Stufenkonzepts, welches die Anpassung der Bereichsgrößen in Abhängigkeit vom aktuellen Patientenaufkommen regelt ( $\triangleright$ Abb. 2 ).
Am 16. März wurden elektive Aufnahmen im Department Innere Medizin gestoppt und elektive Eingriffe im Department Chirurgie und allen anderen operativen Fächern stark reduziert. Die neu geschaffenen COVID-19-Stationen wurden weitestgehend mit Monitorüberwachungen ausgestattet. Weiterhin wurden interdisziplinäre Teams unter Leitung internistischer Fachärzte gebildet und geschult, die unter konsiliarischer Mitbetreuung durch die Infektiologie die Patientenversorgung übernahmen. SOPs zu Diagnostik (inkl. Radiologie) und Therapie auf Normalstationen wurden erstellt. Zudem wurde in Kooperation mit der Klinik für Palliativmedizin ein palliativmedizinisches Betreuungskonzept erarbeitet, das einen eigens vorgesehenen Bettenbereich sowie eine konsiliarärztliche Tätigkeit von Palliativmedizinern auf COVID-19-Stationen vorsieht.

\section{COVID-19-Intensivstationen}

Ende Februar wurde eine Subgruppe zur Prozesssteuerung in der Intensivmedizin initiiert. Ziel war es, die Intensiv- und Beatmungskapazität von vorher regulären 105 Beatmungsbetten substanziell zu erhöhen. Planung und Vorbereitung erfolgten maßgeblich 
durch Mitarbeiter des ARDS (Acute Respiratory Distress Syndrome) -Zentrums, das durch Kooperation der medizinischen und anästhesiologischen Intensivstationen seit Jahren etabliert ist. Mittels Aufrüstung von Normal- zu Intermediate-Care (IMC)Stationen sowie IMC- zu Intensivstationen und konzeptioneller Vorarbeiten zur Umrüstung von OP-nahen Bereichen und Aufwachräumen wurden Voraussetzungen zur Steigerung der Kapazität auf 150 Beatmungsbetten mit Bereichsisolation geschaffen ( $\triangleright$ Abb. 2). Im Ergebnis sollten zusätzliche Therapieplätze mit Erhalt einer möglichst Intensivstations-analogen Behandlungsqualität geschaffen werden. Entsprechend erfolgte eine komplette Herstellung der medizin- und informationstechnischen Therapieplatzausstattung, die über analoge Verfügbarkeiten von Medikamenten, Pflege-/Verbrauchsartikeln und Point-of-care-Diagnostik eine behandlungskompetente Äquivalenz-Infrastruktur für schwerkranke Intensivtherapiepatienten definierte. Ärztliche und pflegerische Expertenteams unter Leitung von speziellen Intensivmedizinern und Intensivtherapiepflegenden erarbeiteten Trainingsprogramme und Simulationen für mehr als 60 Extrabehandlungsplätze. Hierfür wurden Umverteilungen von pflegerischem und ärztlichem Personal von der Normalstation auf die Intensivstation erforderlich. Zur Sicherstellung einer einheitlichen Versorgung wurden interprofessionelle und interdisziplinäre SOPs zur Behandlung erarbeitet und allen Plätzen über eine IT-basierte Kommunikationsstruktur verfügbar gemacht. Zum überregionalen Abgleich der zur Verfügung stehenden Bettenkapazitäten erfolgte ein Austausch zwischen den Intensivstationen auf der Basis einer webbasierten Plattform.

\section{COVID-19 - Klinische Konferenz}

Zur Intensivierung der innerklinischen Kommunikation, zum Erfahrungsaustausch sowie als regelmäßige Fortbildungsveranstaltung mit konzisen Übersichtsvorträgen aus der aktuellen Literatur und Diskussion erarbeiteter SOPs wurde eine klinische COVID-19-Konferenz initiiert. Diese findet seit 25. März 3-mal wöchentlich statt und kann auch im Intranet als Livestream verfolgt werden. In der Konferenz wurden zudem Studienaktivitäten am Universitätsklinikum Freiburg vorgestellt und Studieninitiativen abgestimmt.

\section{Die ersten 115 Patienten in Freiburg}

\section{Epidemiologie, Patientencharakteristiken und Therapie}

Zwischen dem 25.02. und 31.03.2020 wurden 115 Patienten mit PCR-bestätigter SARS-CoV-2-Infektion stationär aufgenommen. Das mediane Alter betrug 64 Jahre, $63 \%$ der Betroffenen waren männlich, 85 Infektionen (74\%) wurden außerhalb der Klinik erworben ( $\triangleright$ Tab. 1). $37 \%$ der Patienten litten unter kardialen Vorerkrankungen (25\% KHK/ischämische Kardiomyopathie, 18 \% Herzinsuffizienz NYHA II-IV), $18 \%$ unter pulmonalen Vorerkrankungen (8\% COPD, $14 \%$ andere chronische Lungenerkrankungen), ein Malignom lag bei $14 \%$ der Patienten, eine primäre oder sekundäre Immundefizienz bei $2 \%$ bzw. $11 \%$ vor. Bei 47 Patienten (41\%) ließ sich keine der genannten Komorbiditäten identifizieren.

Bei Verdacht auf das Vorliegen einer bakteriellen Superinfektion wurden 74 Patienten (64\%) mit einem Antibiotikum (über- wiegend Ampicillin/Sulbactam, bei längerem Krankenhaus-Voraufenthalt Piperacillin/Tazobactam, kein Makrolid-Antibiotikum) behandelt. Eine spezifische antivirale Therapie im Sinne eines individuellen Heilversuchs mit Hydroxychloroquin oder Chloroquin wurde für 5-7 Tage bei 54 Patienten (47\%) eingeleitet, bei 50 Patienten in Kombination mit Lopinavir/Ritonavir. Schwerwiegende kardiale Nebenwirkungen unter Hyroxychloroquin oder Chloroquin im Sinne von höhergradigen Arrhythmien wurden (unter QT-Zeit-Kontrolle) nicht beobachtet. Insgesamt 4 Patienten erhielten eine Anti-IL-6-Therapie mit Tocilizumab. Die Indikation zur Durchführung der Heilversuche wurde im Rahmen infektiologischer Konsile oder Visiten geprüft bzw. gestellt.

\section{Komplikationen und Behandlungsergebnisse}

Insgesamt 37 Patienten (32\%) wurden im Beobachtungszeitraum aufgrund der COVID-19-Erkrankungsschwere intensivstationspflichtig. Patienten aus dieser Gruppe waren älter (Median 68 Jahre), hatten eine höhere Rate an kardialen Vorerkrankungen sowie höhere CRP-, PCT-, IL-6- und Ferritin-Werte im Serum ( $\triangleright$ Tab. 1). Der Anteil der Patienten mit primärer oder sekundärer Immundefizienz oder Chemotherapie war hingegen in der schwerer erkrankten Patientengruppe niedriger.

Von 37 Intensivstation-Patienten wurden 31 (84\%) mit Katecholaminen behandelt. Bei 32 Patienten (86\%) wurde eine invasive oder nichtinvasiv Beatmung begonnen. Von diesen Patienten waren zum Zeitpunkt der Auswertung 3 Patienten extubiert, $23 \mathrm{~Pa}$ tienten weiterhin beatmet und 6 Patienten verstorben. Bei insgesamt 8 Patienten war eine extrakorporale Membranoxygenierung (ECMO) -Versorgung erforderlich. Bei 2 Patienten war bis zum Zeitpunkt der Manuskripterstellung eine ECMO-Explantation möglich (nach 6 bzw. 7 Tagen), 4 Patienten sind weiterhin ECMO-pflichtig, 2 Patienten sind verstorben. Es konnten von den 37 Patienten bislang 7 auf Normalstation verlegt werden (3 sind bereits entlassen), 9 sind verstorben und 21 befinden sich immer noch in intensivmedizinischer Behandlung (mediane Behandlungsdauer dieser Patienten bisher 12 Tage). Bei 35 der 37 Patienten (95\%) wurde eine Antibiotikatherapie durchgeführt. Es zeigte sich kein Fall einer gesicherten invasiven Aspergillose, eine antimykotische Prophylaxe wurde nicht durchgeführt. Insgesamt verstarben bis zum 31.03.2020 17 der 115 Patienten (medianes Alter 83 Jahre), hiervon wurden bei 8 Patienten aufgrund des Patientenwillens keine intensivmedizinischen Maßnahmen eingeleitet.

\section{Diskussion}

Der SARS-CoV-2-Ausbruch stellt das medizinische Versorgungssystem in Deutschland und weltweit auf allen Ebenen vor größte Herausforderungen [3-5]. Aufgrund der eindrücklichen Erfahrungen aus China und Südkorea, der epidemiologischen Daten aus Italien und des späteren, eher punktuellen Ausbruchbeginns war in Deutschland eine längere Vorbereitungszeit zur Entwicklung ambulanter und stationärer COVID-19-Versorgungsmodelle vorhanden.

Als hilfreich für die zügige Aufnahme der Planung und Initiierung der COVID-19-Response stellte sich die bereits vorbestehende Vernetzung der Abteilung Infektiologie, des UNZ und des Insti- 
- Tab. 1 Epidemiologische und klinische Charakteristiken, Therapie und Behandlungsergebnisse von 115 COVID-19-Patienten in Abhängigkeit von der Versorgung auf der Normal- oder Intensivstation.

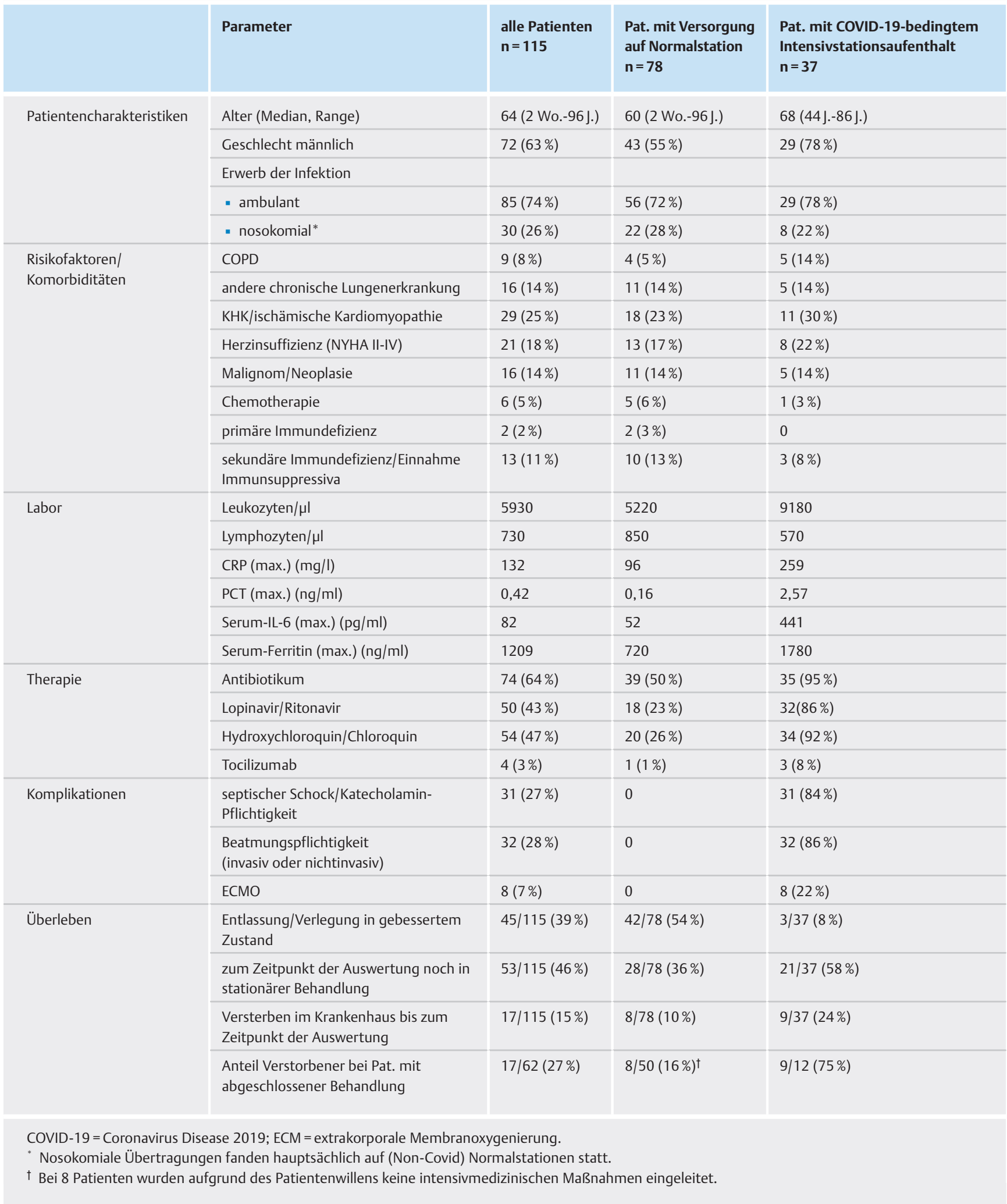


tuts für Krankenhaushygiene - etabliert als Ebola-Taskforce im Jahr 2014 - heraus. Aus dieser Kern-Arbeitsgruppe entwickelte sich unter Einbeziehung weiterer Disziplinen/Abteilungen rasch die Taskforce Coronavirus. Mithilfe der eingesetzten Organisationsstrukturen und des dynamischen Versorgungsmodells konnten u. a. über Schaffung interdisziplinär geführter Stationen Versorgungsengpässe vermieden und sämtliche indizierten und von den Patienten gewünschten Behandlungsmodalitäten unter Freihaltung ausreichender Reservekapazitäten durchgeführt werden. Als Voraussetzungen zur Etablierung des Konzepts dürfen ein hohes Maß an Interdisziplinarität innerhalb des Universitätsklinikums und die frühe und enge Einbindung von außeruniversitären Partnern genannt werden. Dementsprechend fand eine rege Interaktion und enge Absprache mit dem Gesundheitsamt und der Stadt Freiburg, dem Land Baden-Württemberg, der niedergelassenen Ärzteschaft sowie Kassenärztlichen Vereinigung, den umliegenden Kliniken und Rehabilitationseinrichtungen und später mit Trägern von Pflegeeinrichtungen statt.

Eine besondere Herausforderung stellte initial die begrenzte SARS-CoV-2-Testkapazität dar, die trotz Personalaufstockung den Testbedarf nicht gänzlich decken konnten. Aufgrund der unsicheren Versorgungs-/Nachlieferungssituation von Schutzmaterialien und einer ungeklärten Datenlage konnten eskalierte krankenhaushygienische Maßnahmen (z. B. universelles Tragen von Mund-Nasen-Schutz) nicht frühzeitig oder für alle Bereiche empfohlen werden.

Die Durchdringung von festgelegten Abläufen oder Beschlüssen der Taskforce/Klinikeinsatzleitung in den einzelnen Funktionsbereichen, Departments, Kliniken und Instituten gestaltete sich mitunter schwierig, da keine einheitlichen und vordefinierten Meldewege vorhanden waren. So wurde rasch die Bedeutung einer intensiven und weitreichenden Kommunikationsstrategie auch innerhalb des Klinikums klar. Um dem aktiv zu begegnen wurden 3-mal wöchentlich Sitzungen des Departments für Innere Medizin sowie klinische COVID-19-Konferenzen, die als zentrale Fortbildungsveranstaltung zusätzlich dienten, eingerichtet. Des Weiteren erfolgten tägliche Regelkommunikationen zu den Operations- und Interventionskapazitäten sowie regelhafte LiveStream-Klinikdirektoren-Konferenzen vonseiten des Vorstands.

Der klinische Verlauf der COVID-19-Patienten entsprach den bisher publizierten Beschreibungen [6-8]. Die Subgruppe der schwererkrankten Patienten entwickelte eine respiratorische Insuffizienz oftmals gegen Ende der ersten Erkrankungswoche. Fortgeschrittenes Alter und kardiopulmonale Grunderkrankungen waren Prädiktoren für einen schwereren Verlauf mit erforderlichem Intensivstationsaufenthalt [9]. Es deutet sich an, dass das Vorliegen einer primären oder sekundären Immundefizienz (medikamentöse Immunsuppression, Chemotherapie) nicht in gleichem Maße für einen schwerwiegenderen Verlauf prädisponiert bzw. in Subgruppen ggf. mit einem minderschweren Verlauf assoziiert sein könnte. Für valide Aussagen sind jedoch größere Kohorten und längere Beobachtungszeiträume erforderlich. Gleiches gilt für den Einfluss antiviraler oder antientzündlicher Therapiestrategien [10-12]. Die Krankenhaussterblichkeit ist mit 15\% vergleichsweise niedrig, jedoch noch nicht repräsentativ, da unge- fähr die Hälfte der noch hospitalisierten Patienten in kritischem Zustand auf Intensivstationen weiterversorgt wird. Auch unter früher Verfügbarkeit sämtlicher intensivmedizinischer Möglichkeiten inkl. extrakorporaler Lungenersatzverfahren stellen sich die protrahierten Beatmungstherapien als besonders herausfordernd dar. Invasive Beatmungstherapien und maximale Lagerungstherapien, häufige konkomitierende Nierenersatztherapien sowie der vermehrt notwendige Einsatz von ECMO-Therapien resultieren bei den schwersterkrankten COVID-19-Patienten mit einem Acute Respiratory Distress Syndrome (ARDS) in Intensivstationsaufenthalten von zumeist mehr als 2-3 Wochen.

\section{Schlussfolgerung (und Ausblick)}

Durch die beschriebenen Maßnahmen, die durch Zusammenwirken sämtlicher Disziplinen, Abteilungen und Geschäftsbereiche des Universitätsklinikums Freiburg geschultert werden, konnte eine adäquate Versorgung von COVID-19-Patienten sichergestellt und die Handlungsfähigkeit des Klinikums in der aktuellen Ausbruchsituation erhalten werden. Die wirtschaftlichen Konsequenzen für das Universitätsklinikum Freiburg sowie eventuelle Auswirkungen auf die Versorgung von Nicht-COVID-19-Erkrankten dürfen in diesem Kontext nicht außer Acht gelassen werden und sollten in zukünftigen Studien erfasst werden.

\section{KERNAUSSAGEN}

- Frühe Aktivitäten und Impulse zur COVID-19-Versorgung kamen von einer im Rahmen des Ebola-Ausbruchs 2014 formierten Arbeitsgruppe, um die sich rasch eine Taskforce Coronavirus bildete - eine Verstetigung derartiger Aktivitäten bzw. Arbeitsgruppen erscheint ratsam.

- Der Ausbau der ambulanten Testung und Beratung, die Separierung der Patientenströme sowie die Eskalation der Versorgungskapazitäten auf Normal- und Intensivstationen waren elementare Maßnahmen in der entwickelten COVID-19-Versorgung.

- Die Anpassung von Organisationsstrukturen, ein hohes $\mathrm{Maß}$ an Interdisziplinarität und eine enge Einbindung außeruniversitärer Partner waren Voraussetzungen zur Schaffung eines dynamischen Versorgungsmodells.

- Das erarbeitete Modell sicherte eine adäquate Versorgung von COVID-19-Patienten und den Erhalt der Handlungsfähigkeit in der aktuellen Ausbruchsituation - die wirtschaftlichen Konsequenzen für das Universitätsklinikum Freiburg und eventuelle Auswirkungen auf die Versorgung Nicht-COVID-19-Erkrankter sollten in zukünftigen Studien erfasst werden.

- Höheres Alter und kardiopulmonale Komorbiditäten prädisponieren zu schwerwiegenden Verläufen mit protrahierter Beatmungs- und Intensivstationspflichtigkeit sowie hoher Mortalität. 
Interessenkonflikt

Die Autorinnen/Autoren geben an, dass kein Interessenkonflikt besteht.

\section{Literatur}

[1] European Centre for Disease Prevention and Control. Coronavirus disease 2019 (COVID-19) in the EU/EEA and the UK - eighth update (08. April 2020). Im Internet (Stand: 23. April 2020): https://www.ecdc.europa.eu/ en/publications-data/rapid-risk-assessment-coronavirus-disease-2019covid-19-pandemic-eighth-update

[2] Robert-Koch-Institut. Aktueller Lage-/ Situationsbericht des RKI zu COVID-19. https://www.rki.de/DE/Content/InfAZ/N/Neuartiges_Corona virus/Situationsberichte/Gesamt.html; Stand: 23.4.2020

[3] Armocida B, Formenti B, Ussai S et al. The Italian health system and the COVID-19 challenge. Lancet Public Health 2020 Mar 25

[4] European Centre for Disease Prevention and Control. Guidance for health system contingency planning during widespread transmission of SARSCoV-2 with high impact on healthcare services (März 2020). Im Internet (Stand: 23.4.2020): https://www.ecdc.europa.eu/sites/default/files/docu ments/COVID-19-guidance-health-systems-contingency-planning.pdf

[5] Liew MF, Siow WT, MacLaren G et al. Preparing for COVID-19: early experience from an intensive care unit in Singapore. Crit Care 2020; 24: 83. doi:10.1186/s13054-020-2814-x
[6] Guan W], Ni ZY, Hu Y et al. Clinical Characteristics of Coronavirus Disease 2019 in China. N Engl J Med 2020. doi:10.1056/NEJMoa2002032

[7] Zhou F, Yu T, Du R et al. Clinical course and risk factors for mortality of adult inpatients with COVID-19 in Wuhan, China: a retrospective cohort study. Lancet 2020; 395: 1054-1062

[8] Schilling J, Diercke M, Altmann D et al. Vorläufige Bewertung der Krankheitsschwere von COVID-19 in Deutschland basierend auf übermittelten Fällen gemäß Infektionsschutzgesetz. Epid Bull 2020; 17: 3-9. doi: $10.25646 / 6670$

[9] Chow N, Fleming-Dutra K, Gierke R. Preliminary Estimates of the Prevalence of Selected Underlying Health Conditions Among Patients with Coronavirus Disease 2019 - United States, February 12-March 28, 2020. MMWR Morb Mortal Wkly Rep 2020; 69: 382-386. doi:http:// dx.doi.org/10.15585/mmwr.mm6913e2

[10] Cao B, Wang Y, Wen D et al. A Trial of Lopinavir-Ritonavir in Adults Hospitalized with Severe Covid-19. N Engl ] Med 2020. doi:10.1056/ NEJMoa2001282

[11] Grein J, Ohmagari N, Shin D et al. Compassionate Use of Remdesivir for Patients with Severe Covid-19. N Engl J Med 2020. doi:10.1056/ NEJMoa2007016

[12] Ritchie AI, Singanayagam A. Immunosuppression for hyperinflammation in COVID-19: a double-edged sword? Lancet 2020; 395: 1111 